\title{
Flossing bands to treat keinböck's disease in a collegiate men's basketball player: a case report
}

\begin{abstract}
Background: Keinböck's disease (KD) is an uncommon medical condition characterized by avascular necrosis of the lunate. Conservative treatment of this condition consists of immobilization, strengthening and range of motion (ROM) exercises of the surrounding musculature of the wrist. Tissue flossing is a relatively new treatment technique that incorporates wrapping latex bands around the targeted body part, followed by a brief period of exercise. Tissue flossing is thought to provide similar therapeutic effects to blood reperfusion and/or blood flow restriction therapy.
\end{abstract}

Purpose: Present a clinical case detailing the use of flossing bands in the treatment of a collegiate men's basketball player suffering from $\mathrm{KD}$ of the right wrist.

Treatment: A 21-year old basketball player reported to the athletic training clinic complaining of worsening wrist pain and stiffness. The patient had attempted conservative treatment with little relief. Following evaluation with magnetic resonance imaging, the patient was diagnosed with KD. At this time, the use of tissue flossing to attempt to restore pain free ROM and to potentially increase blood flow began. After two weeks of treatment, the patient's symptoms and reported function had improved. The patient continued treatment and rehabilitation program until the beginning of his competitive season, at which point he began a maintenance program.

Uniqueness: The KD diagnosis would increase likelihood of surgical intervention later on. However, in this case, the patient was able to return to full function without surgical intervention to date. We are unaware of any case report describing the use of tissue flossing bands as an adjunct treatment for $\mathrm{KD}$.

Conclusion: Tissue flossing bands may be a viable therapeutic procedure for symptoms such as pain and/or decreased ROM. Further studies need to be conducted in order to determine the potential therapeutic application and methodology of tissue following bands.

Keywords: keinböck's disease, lunatomalacia, flossing bands
Volume 3 Issue 2 - 2018

\author{
S Andrew Cage, ${ }^{1,2}$ Brandon JWarner, ${ }^{2,3}$ Phil \\ Stevenson, ${ }^{4}$ Arturo A Arce-Esquivel' \\ 'Department of Health and Kinesiology, The University of Texas \\ at Tyler, USA \\ ${ }^{2}$ Kinesiology Department, University of North Carolina at \\ Greensboro, USA \\ ${ }^{3}$ Grand Canyon University, USA \\ ${ }^{4}$ University of Saint Joseph, USA
}

\begin{abstract}
Correspondence: Arturo A.Arce-Esquivel, M.D., Ph.D. Department of Health and Kinesiology, College of Nursing and Health Studies, The University of Texas at Tyler, USA, Tel (903) 565-5838; Fax (903) 566-7065, Email aarce@uttyler.edu
\end{abstract}

Received: March 30, 2018 | Published: April 23, 2018

\section{Introduction}

Keinböck's disease (KD) is defined as ischemia and necrosis of the lunate bone that occurs in the absence of an acute fracture or nonunion fracture. ${ }^{1}$ Presently, the exact etiology of KD is unknown, but is postulated to be secondary to repetitive or acute trauma to the wrist resulting in damage to the blood supply to the lunate. ${ }^{2}$ In the absence of a definitive mechanism for $\mathrm{KD}$, possible predispositions include; shape and size of the lunate, and vascularity of the lunate. ${ }^{3}$ Clinical presentation of KD has been associated with swelling and pain over the lunate, loss of grip strength, weakness with ulnar deviation, and pain with passive third finger extension. ${ }^{4}$ Standard conservative treatment of $\mathrm{KD}$ consists of immobilization of the joint then progressing to range of motion (ROM) and strengthening exercises of the surrounding musculature. ${ }^{1,5}$ In the event that conservative treatment fails, surgical interventions may include; vascular grafting, radial shortening, ulnar lengthening, proximal row carpectomy, and decompression osteotomies. ${ }^{1,5}$ Unfortunately, these present surgical options are often termed "salvage procedures". If a patient is forced to resort to surgery, the prognosis for returning to high level physical activity is poor.

Tissue flossing is a relatively new treatment technique incorporating the use of latex bands to wrap the pathologic area prior to a brief bout of exercise. ${ }^{6,7}$ Though available research is lacking, anecdotal evidence suggests that tissue flossing may be a viable method of increasing joint ROM, decreasing pain, and improving athletic performance.? One proposed mode of action for tissue flossing is similar to the tissue reperfusion that occurs with blood flow restriction therapy. ${ }^{7}$ However, recent studies have not found data to suggest that tissue flossing has a significant effect on regional blood flow. ${ }^{6}$ Despite evidence of a known mechanism, few studies suggest tissue flossing may improve joint ROM, perceived ability to move a joint through space, and potential increased torque generation at the targeted joint. ${ }^{6-8}$

Due to lack of research, there is an absence of standardized methodology for the use and application of flossing bands. When applying flossing bands, clinicians must rely on anecdotal evidence or previous experience when determining treatment parameters. Even though flossing bands possess numerous limitations, the potential and anecdotally reported positive patient outcomes warrant further study to determine best clinical practices with regards to application and treatment parameters.

The aim of this case report is to document the treatment of a patient suffering from $\mathrm{KD}$ of the right wrist. Upon confirmed diagnosis, the patient was treated using flossing bands in addition to wrist strengthening and ROM exercises. The present case report includes background information on KD and tissue flossing, descriptions of the treatment and rehabilitation process, and the subsequent successful return to participation. 


\section{Case report}

A 21-year-old men's collegiate basketball player reported to the athletic training clinic complaining of pain and swelling over the dorsal aspect of his right wrist. In addition, the patient also reported stiffness and perceived weakness with all wrist motions. Patient indicated a previous history of a fractured bone in his wrist when he was a freshman in high school, but could not recall which bone was injured. Following his clearance from his fracture, the patient began to experience an insidious onset of increasing pain and stiffness in his wrist. After the patient's first year at his current institution, all wrist related symptoms had either worsened or remained constant with conservative treatment, which included; (a) cryotherapy, (b) paraffin bath, and (c) therapeutic exercise aimed at strength and ROM restoration in the wrist.

With documented failure of conservative treatment while returning to participation, the patient was referred to the team physician for further evaluation. Based off of the severity of the patient's symptoms, the team physician ordered an MRI to assess for scapholunate dissociation. The MRI revealed the patient's lunate had undergone a vascular necrosis consistent with KD (Figure 1). It was determined, in consultation with two additional hand surgeons, that the patient's lunate was likely not salvageable. The prognoses for a non-surgical recovery, as well as surgical interventions were discussed with the patient. The patient elected to attempt non-operative treatment and rehabilitation with the athletic training staff in order to participate in his final season of competitive basketball.

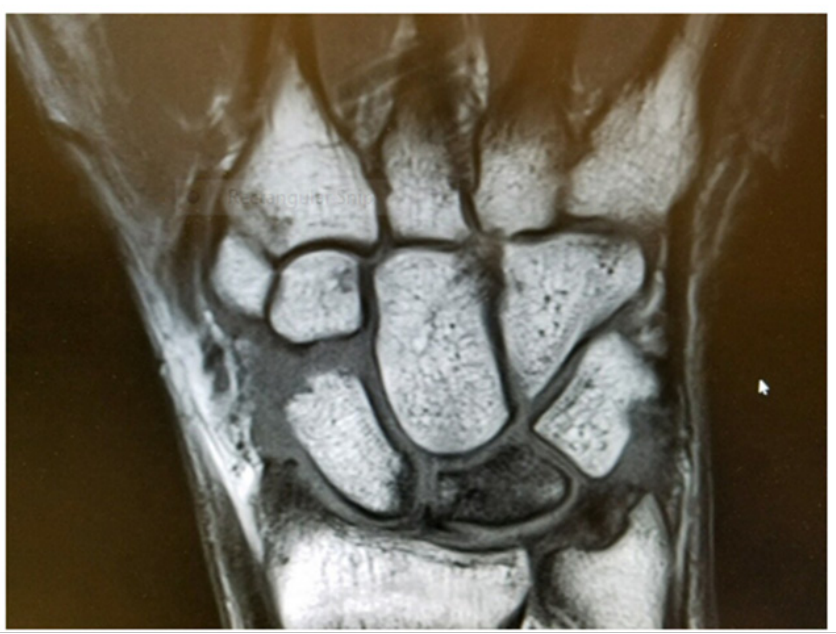

Figure I Magnetic resonance image of the patient's right wrist showing avascular necrosis of the lunate.

After consultation with the team physician and hand surgeons to determine the appropriate non-operative protocol, the patient began rehabilitation design to improve wrist strength and ROM in all directions. Before the patient's first treatment and rehabilitation session, the patient filled out a Wrist/Hand Disability Index (WHDI) and visual analog scale (VAS) to determine the extent of dysfunction and pain related to his wrist pathology. ${ }^{9}$ The WHDI consisted of a series of questions, divided in 10 sections (e.g., pain intensity, numbness and tingling, driving, work, etc.), asking the patient to rate the extent to which his wrist pain would limit various activities of daily living (ADL). ${ }^{9}$ The WHDI scores for each section goes from 1 to
5; where $1=$ great limitation, and $5=$ no limitations for ADL. The VAS used to assess the patient's wrist pain consisted of an unmarked 10 $\mathrm{cm}$ line with the left end of the line indicating no pain at all $(=0 \mathrm{~cm})$, and the right end of the line indicating worst pain possible $(=10 \mathrm{~cm})$. The patient was asked to place a mark on the line corresponding to the amount of pain he felt at the time of assessment.

The patient performed these measures on a weekly basis, over a 6-week period, to determine the need to discontinue or modify interventions. Prior to the beginning of therapeutic exercise, the patient underwent tissue flossing. This treatment consisted of wrapping a 100\% natural latex band (Rock Floss, Rock Tape, Campbell, CA) through the web space of the wrist and around the distal wrist. Following placement of the band, a clinician would apply tension to the band using their hands for one to three minutes based off of patient tolerance (Figure 2). Next, the patient would rotate their wrist clockwise and counterclockwise 20 times in each direction. Following completion of the wrist rotation, the band was removed and the patient began his exercise program.

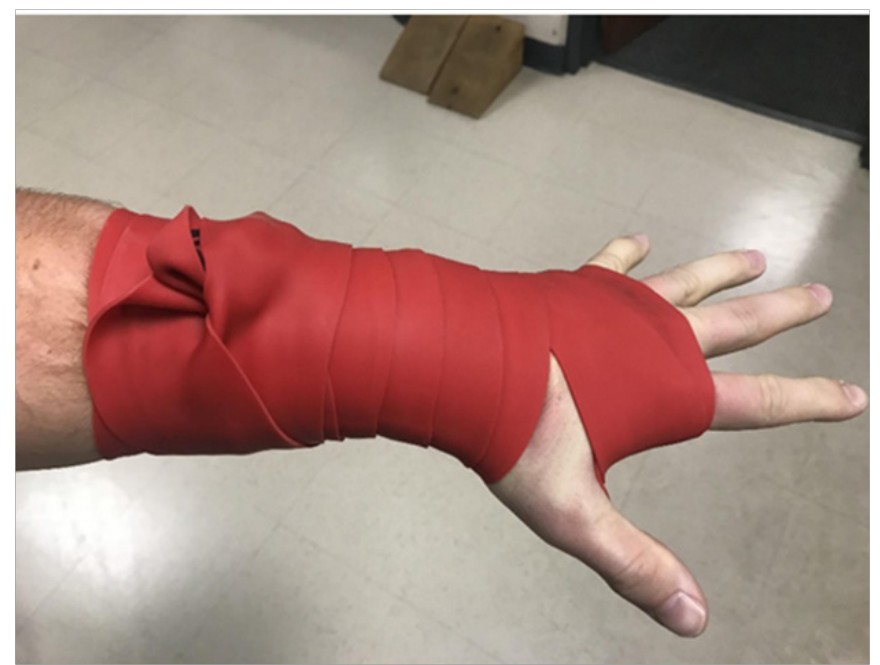

Figure 2 Wrapping technique for flossing bands utilized in treatment program.

Throughout the remainder of the patient's competitive season, the patient continued with the previously described treatment and rehabilitation regimen. The patient repeatedly indicated his satisfaction with his increased strength and ROM of his wrist. Given this improvement in function and decrease in symptoms, the patient stated his intent to delay surgery so long as conservative treatment measures were providing substantial relief.

\section{Results}

Over the 6-week of tissue flossing treatment, the WHDI increased significantly by $45 \%$ (Table 1 ), from 3.3 (week 1 ) to 4.8 (week 6 ). The VAS decreased significantly by $88 \%$, from $5.8 \mathrm{~cm}$ (week 1 ) to $0.7 \mathrm{~cm}$ (week 6). The differences in WHDI across the 6 weeks were examined using analysis of variance with repeated measures. Whilst, the VAS, week 1 and week 6 difference, was examined using independent t-test. The statistical analyses were performed with Graph Pad Prism version 6.0a (Graph Pad Software, Inc. CA, USA). The level of significance was set at $\mathrm{p}<0.05$ 
Table I Functional assessment

\begin{tabular}{lllllll}
\hline Variable & Week I & Week 2 & Week 3 & Week 4 & Week 5 & Week 6 \\
\hline Average WHDI (absolute units) & $3.3 \pm 1.6$ & $4.1 \pm 0.3 *$ & $4.6 \pm 0.52 *$ & $4.7 \pm 0.48 *$ & $4.8 \pm 0.42 *$ & $4.8 \pm 0.42 *$ \\
\hline
\end{tabular}

WHDI,Wrist Hand Disability Index; Values are mean \pm SD. ${ }^{*} \mathrm{p}<0.05$ as compared to week I.

\section{Discussion}

$\mathrm{KD}$ is a relatively rare pathology affecting the lunate as well as general wrist strength and ROM. ${ }^{1}$ While the majority of the cases of $\mathrm{KD}$ result in marked pain, swelling, tenderness, and loss of function specific to the dorsal aspect of the wrist, as many as $1.9 \%$ of cases can remain asymptomatic. ${ }^{4,10}$ Early diagnosis of $\mathrm{KD}$ is crucial if a patient and clinician hope to avoid surgical intervention. ${ }^{1}$ However, there is no consensus on the threshold for electing to undergo surgical intervention, and which intervention will yield the best patient outcomes. ${ }^{10}$ Because of this, treatment options are chosen largely based on patient values and caregiver experience and expertise. ${ }^{11}$

This case report describes the diagnosis, and subsequent treatment and rehabilitation of a patient suffering from KD. In this case, the patient experienced documented relief of symptoms with the use of tissue flossing in conjunction with therapeutic exercise to address strength and ROM deficits. The delays in diagnosis of the patient's pathology make it difficult to determine whether or not a vascular necrosis would have been avoidable with conservative or surgical intervention. However, it is still noteworthy that the patient was able to return to athletic competition using conservative measures given the severity of his condition.

\section{Conclusion}

The field of therapeutic interventions is ever changing as more research is conducted and disseminated to the professional population. It is important for clinicians to remain abreast of new developments and practices that occur within the field of therapeutic intervention in order to place themselves in the best possible position to achieve optimal patient related outcomes. This case report argues, by clinical experience, that the use of tissue flossing bands may present the potential to be an effective therapeutic procedure in cases of KD. Clinicians who choose to incorporate tissue flossing should be sufficiently familiar with the technique and review the pertinent literature that guides the application of the treatment.

While the patient, in this case, received apparent positive outcomes, it is important to note that further research needs to be performed to determine the specific mechanisms and effects of tissue flossing. Until such research can be conducted, with the knowledge that minimal adverse issues have been reported, tissue flossing is a safe treatment method to utilize when applied by a well versed clinician. ${ }^{5}$ When using tissue flossing, clinicians should regularly check their patient's distal blood flow to ensure that the treatment session does not need to be discontinued earlier than planned. ${ }^{5}$ Further research is needed to determine best practices for application and duration of tissue flossing, as well as the optimal phase of healing during which to initiate treatment.

\section{Acknoweledgements}

None.

\section{Conflict of interest}

Authors declare that there are no conflicts of interest.

\section{References}

1. Moron M, Oellig F, Sanchez T. Proximal row carpectomy for coexisting Kienbock's Disease and giant intraosseous ganglion of the scaphoid: a case report and review of the literature. Case Reports in Orthopedics. 2014.

2. Wagner JP, Chung KC. A historical report on Robert Kienbock (18711953) and Kienbock's disease. J Hand Surg. 2005;30:1117-1121.

3. Allan CH, Lichtman DM. Kienböck's Disease: Diagnosis and Treatment: Journal of the American Academy of Orthopedic Surgeons. 2001;9(2):128-136.

4. Starkey C. Sara B. Wrist, Hand, and Finger Pathologies. In Examination of Orthopedic \& Athletic Injuries. 4th ed. Philadelphia, PA: FA. Davis Company.

5. Shah FA, Alam W, Hayat S, et al. Keinbock's Disease: metaphysical core decompression of distal radius-a novel technique to treat.

6. Bohlen J, Arsenault M, Deane B, et al. Effects of applying floss bands on regional blood flow. International Journal of Exercise Science: Conference Proceedings. 2014;9(2).

7. Driller MW, Overmayer RG. The effects of tissue flossing on ankle range of motion and jump performance. Physical Therapy in Sport. 2017;25:20-24.

8. Driller M, Mackay K, Mills B, et al. Tissue flossing on ankle range of motion, jump and sprint performance: a follow-up study. Physical Therapy in Sport. 2017;28:29-33.

9. http://www.dmv-smi.com/wp-content/uploads/whdi.pdf

10. Mennen U, Sithebe H. The incidence of asymptomatic Kienbock's disease. Journal of Hand Surgery: European Volume. 2009;15(1):348350 .

11. Dungen SV, Dury M, Foucher G, et al. Conservative treatment versus scaphotrapeziotrapezoid arthrodesis for Kienbock's disease. A retrospective study. Chir Main. 2006;25:141-45. 